\title{
openheart Genetic variation at the human connexin 43 locus but not at the connexin 40 locus is associated with left bundle branch block
}

\author{
Per Ladenvall, ${ }^{1}$ Björn Andersson, ${ }^{2}$ Mikael Dellborg, ${ }^{1}$ Per-Olof Hansson, ${ }^{1}$ \\ Henry Eriksson, ${ }^{1}$ Dag Thelle, ${ }^{3}$ Peter Eriksson ${ }^{1}$
}

To cite: Ladenvall $P$, Andersson B, Dellborg M, et al. Genetic variation at the human connexin 43 locus but not at the connexin 40 locus is associated with left bundle branch block. Open Heart 2015;2:e000187. doi:10.1136/openhrt-2014000187

Received 14 August 2014 Revised 11 November 2014 Accepted 20 January 2015

\section{(a) CrossMark}

${ }^{1}$ Department of Molecular and Clinical Medicine/ Cardiology, Institute of Medicine, Sahlgrenska Academy at University of Gothenburg, Gothenburg, Sweden

${ }^{2}$ Department of Pediatrics, Göteborg Pediatric Growth Research Center, Institute of Clinical Sciences,

Sahlgrenska Academy at University of Gothenburg, Gothenburg, Sweden ${ }^{3}$ Department of Community Medicine and Public Health, Institute of Medicine, Sahlgrenska Academy at University of Gothenburg, Gothenburg, Sweden

Correspondence to Dr Per Ladenvall; per.ladenvall@gu.se

\section{ABSTRACT}

Background: Bundle branch block (BBB) has been regarded as a disease of the conduction system, but occurs in mice lacking connexin 40 (expressed in atria, proximal conduction system) or connexin 43 (expressed in Purkinje cells, cardiomyocytes).

Objective: The aim of this paper is to explore whether $\mathrm{BBB}$ is heritable, and whether polymorphisms at connexin 40 and connexin 43 loci are associated with BBB.

Methods: To assess BBB heritability, we screened descendants of men with BBB in the population cohort 'The Study of Men Born 1913'. DNA samples from 80year-old men with extreme QRS-duration phenotypes were used to search for polymorphisms at connexin 40 and 43 loci. Associations between identified polymorphisms and BBB were evaluated in an independent cohort (INTERGENE).

Results: Seventy-seven men from 'The Study of Men Born 1913' with BBB had 116 descendants. Among the 76 participating descendants, 2 sons $(6.4 \%)$ had BBB at 54 years of age. At the same age, $0.9 \%$ of men born in 1913 had BBB. We identified 6 single nucleotide polymorphisms (SNPs) in connexin 40 and 1 polymorphism in connexin 43. In the INTERGENE cohort, the connexin 43 polymorphism was associated with left BBB (LBBB) (4 of 35 LBBB vs 16 of 232 without $B B B, \chi^{2}=7.4, p=0.03$ ), but not with right $B B B$ (RBBB) or overall BBB. None of the connexin 40 SNPs or haplotypes were associated with LBBB or RBBB.

Conclusions: These findings indicate that conduction by connexin 43 within the ventricular muscle distal to the specialised conduction system may be important for LBBB development.

\section{INTRODUCTION}

The clinical implications of bundle branch block (BBB) have long been a subject of dispute. ${ }^{1}$ The ECG alone does not allow us to determine whether the conduction disturbance is a function of a focal lesion in main bundle branches or the result of a more diffuse myocardial disease producing a type of parietal block. ${ }^{12}$

\section{KEY MESSAGES}

What is already known about this subject?

- Bundle branch block is associated with an increased risk of sudden cardiac death. Reduced levels of connexin 40 are associated with bundle branch block and reduced levels of connexin 43 are associated with increased risk of ventricular arrhythmias.

What does this study add?

- The present study indicates that bundle branch block might be due to a diffuse myocardial process within the myocardium and not only due to ischaemia of the specialised conduction system. It also indicated that there might be a genetic susceptibility for bundle branch block development.

How might this impact on clinical practice?

- This might lead to an increased awareness that bundle branch block could be due to a diffuse myocardial process and thereby stimulate further research in this field. This further research might identify responders to cardiac resynchronisation therapy (CRT) more easily.

In a previous study of a Swedish general population sample of men followed from 50 to 80 years of age, The Study of Men Born 1913 , we found $86 \%$ of the BBB to be acquired and not correlated to ischaemic heart disease or its risk factors. ${ }^{3}$ Similar findings have been observed in a large Finnish data set of more than 10000 participants, ${ }^{4}$ whereas in the US Framingham data set, the majority of BBB found was associated with cardiovascular disease or its risk factors. ${ }^{5} 6$ These results support the theory that BBB is a marker of a progressive disease that also affects conduction within the myocardium. ${ }^{34}$

The development of cardiac resynchronisation therapy for patients with BBB and heart failure has stimulated research on $\mathrm{BBB},{ }^{7} 8$ but the underlying nature of this progressive 
disorder is still mostly unknown. It is also unsolved whether BBB should be regarded as a single phenotype or if right BBB (RBBB) and left BBB (LBBB) are different entities. Therefore, we analysed all three, BBB, RBBB and LBBB, in the present study.

Molecular studies on animal and on human data have shown that variation in connexin 40 and connexin 43 can cause BBB and is associated with cardiomyopathy. ${ }^{9-11}$ Connexin 40 is expressed in the atria and in the proximal parts of the conduction system, coexpressed with connexin 43 in Purkinje fibres, and mice lacking connexin 40 have prolonged PQ interval, AV interval and QRS duration due to reduced conduction within the Purkinje fibres. ${ }^{12}$ It has also been shown to produce a phenotype of RBBB. ${ }^{13}$ In contrast, connexin 43 is expressed in the Purkinje fibres and working cardiomyocytes. In heterozygous knock-out mice with reduced connexin 43 levels, ventricular conduction was markedly reduced and had increased risk of ventricular arrhythmias. ${ }^{14}$ Moreover, human studies on heart failure have shown that connexin 43 expression is reduced in end-stage heart failure, ${ }^{10}$ and this altered expression is associated with an increased risk of ventricular arrhythmias. ${ }^{11}$

The hypothesis for the present study is that there is a hereditary predisposition to acquire BBB and that predisposition is associated with polymorphisms in genes coding for connexin 40 and connexin 43. This hypothesis is tested in three successive steps:

- Heredity of the ECG phenotype BBB or prolonged QRS duration is tested in the descendants of men with BBB from the population sample of The Study of Men Born 1913.

- Genetic variation at the connexin 40 and connexin 43 loci is explored by genotyping samples from The Study of Men Born 1913 with and without BBB.

- The association of identified polymorphisms with BBB is subsequently tested in the independent INTERGENE sample.

\section{METHODS}

Three samples for testing the three different steps of our hypothesis were used. The relation between populations for the analysis of heredity and the resequencing analysis is depicted in figure 1 . The association analysis was performed in an independent sample as described below.

\section{Patients for step 1: heredity-The Study of Men Born 1913 and their descendants}

The Study of Men Born 1913 is a randomly selected general population of men followed since $1963 .{ }^{15}$ For this paper, we use data from 1963 until 1993, when 361 men (then aged 80 years) were still alive; 212 (59\%) participated in the follow-up examination. Among the participating men, a diagnosis of $\mathrm{BBB}$ was made in 82 patients during 30 years of follow-up: 60 with RBBB and 22 with LBBB, as previously reported. ${ }^{3}$ The majority $(86 \%)$ had acquired BBB unrelated to ischaemic heart

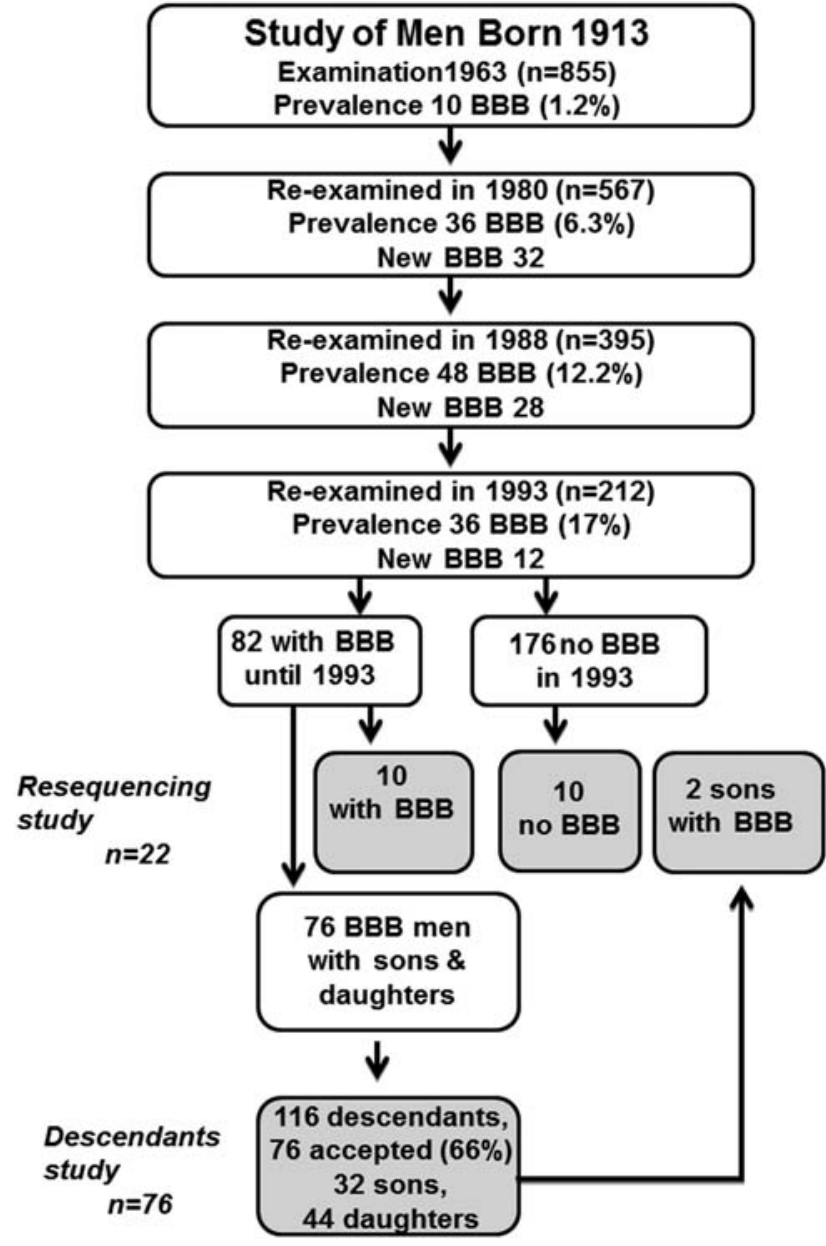

Figure 1 Relation between the study populations in the resequencing and the descendants investigations (the descendants study) (BBB, bundle branch block).

disease. Of these 82 men, we were able to construct pedigrees in 77 of the men's families; the families of the remaining 5 men could not be traced. The 77 men had 116 sons and daughters, all of whom were invited to an investigation including physical examination, standardised history forms, spirometry and 12-lead ECG recording. Of the 116 descendants, $76(66 \%)$ were willing to participate and they formed the basis of the report on the ECG phenotype (table 1).

\section{Patients for step 2: resequencing}

In order to separate the ECG phenotype optimally for the genetic analysis, we chose two groups from The Study of Men Born 1913. ${ }^{3}$ The first group consisted of 10 men with BBB (8 had RBBB and 2 had LBBB) who had the longest QRS duration from the youngest age and the second group was a contrast group of 10 men at age 80 without BBB and with the shortest possible QRS duration. The 10 men with BBB had a mean QRS duration of $136 \mathrm{~ms}$ that was present at the mean age of 63.5 years. The 10 men in the contrast group had a mean QRS duration of $78 \mathrm{~ms}$ and a mean age of 80 years. In addition to these 20 participants, 2 descendants identified as 
Table 1 Baseline characteristics and 12-lead ECG phenotype in descendants of men born in 1913 with bundle branch block

\begin{tabular}{|c|c|c|c|}
\hline & All $(n=76)$ & Sons $(n=32)$ & Daughters $(n=44)$ \\
\hline Mean age, years & $54 \pm 5$ & $55 \pm 6$ & $54 \pm 5$ \\
\hline Length, $\mathrm{cm}$ & $171 \pm 9$ & $179 \pm 6$ & $165 \pm 6$ \\
\hline Weight, kg & $78 \pm 14$ & $86 \pm 10$ & $72 \pm 13$ \\
\hline $\mathrm{BMI}$ & $26.4 \pm 3.8$ & $26.8 \pm 2.8$ & $26.2 \pm 4.4$ \\
\hline Systolic blood pressure, mm Hg & $130 \pm 18$ & $132 \pm 16$ & $129 \pm 18$ \\
\hline Diastolic blood pressure, $\mathrm{mm} \mathrm{Hg}$ & $80 \pm 9$ & $81 \pm 9$ & $78 \pm 8$ \\
\hline Heart rate, bpm & $69 \pm 11$ & $67 \pm 13$ & $70 \pm 10$ \\
\hline Spirometry, peak flow, mL/s & $524 \pm 114$ & $621 \pm 85$ & $450 \pm 68$ \\
\hline Sokolow-Lyon, mm & $25 \pm 6$ & $28 \pm 7$ & $24 \pm 5$ \\
\hline$P Q$ duration, ms & $155 \pm 19$ & $160 \pm 21$ & $152 \pm 17$ \\
\hline QTc, ms & $410 \pm 21$ & $409 \pm 22$ & $411 \pm 21$ \\
\hline $\mathrm{P}$ duration, ms & $110 \pm 12$ & $111 \pm 12$ & $109 \pm 12$ \\
\hline QRS duration, ms & $92 \pm 12$ & $97 \pm 16$ & $88 \pm 7$ \\
\hline Complete bundle branch block, $\mathrm{n}$ & 2 & 2 & 0 \\
\hline
\end{tabular}

Continuous variables are expressed as mean \pm SEM.

$\mathrm{BMI}$, body mass index.

having BBB, 1 with $\mathrm{LBBB}$ and 1 with $\mathrm{RBBB}$, were included in the screening panel, resulting in 10 participants without BBB and 12 with BBB.

\section{Patients for step 3: association}

In total, 3600 participants aged 25-70 years were randomly sampled from individuals living in Västra Götalands region as controls into the INTERGENE study cohort. Clinical examinations, ECG and collection of blood samples for DNA analysis were carried out as described. ${ }^{16}$ Out of the INTERGENE study cohort, 121 participants had an ECG indicating a possible BBB (QRS over $120 \mathrm{~ms}$ ) and an adequate blood sample for DNA analysis. ECG classification by two cardiologists (see $E C G$ below) resulted in 108 participants with BBB for the present study, 35 with LBBB and 73 with RBBB. For each case with possible BBB, 2 controls from the population cohort without clinically overt cardiovascular disease and without BBB, matched for age and sex, were selected (table 2).

\section{Genetic analysis-resequencing}

DNA was extracted from whole blood (if available) or serum. The screening panel was studied by resequencing by di-deoxy sequencing, as described elsewhere. ${ }^{17}$ A screening panel of 44 chromosomes resulted in $99 \%$ power to find any biallelic polymorphism with an allelic frequency of $10 \%$, and $90 \%$ power for polymorphisms with an allelic frequency of $5 \%$. For the connexin 40 gene, all described exons were analysed, as well as 1200 bp of the promoter; for the connexin 43 gene, all exons as well as $1000 \mathrm{bp}$ of the promoter were sequenced.

\section{Genetic analysis-genotyping}

Genotyping was performed by TaqMan assavs on the 7900 HT system (Applied Biosystems, Life Technologies, New York, USA). Primer and probe data are available from the authors on request.

\section{ECG}

Standard 12-lead ECGs were recorded with participants at rest in the supine position. Paper speed was $50 \mathrm{~mm} / \mathrm{s}$, and calibration was $1 \mathrm{mV}: 10 \mathrm{~mm}$. ECGs with a QRS duration above $120 \mathrm{~ms}$ were considered for the study. Two independent cardiologists (PE and MD), classified the ECGs as RBBB, LBBB or not BBB (including hypertrophy and Wolff-Parkinson-White syndrome, which were excluded from further analyses). LBBB was defined as QRS duration above $120 \mathrm{~ms}$, PQ interval above $120 \mathrm{~ms}$, upright and slurred R-waves in leads I, V5, V6 and QS, or rS pattern in lead V1. ${ }^{18}$ The only deviation from the interpretation proposed by $\mathrm{AHA}^{19}$ is that we also included LBBB with a small $\mathrm{q}$ in leads V5 and V6. $\mathrm{RBBB}^{20}$ was defined as QRS duration above $120 \mathrm{~ms}$, PQ interval above $120 \mathrm{~ms}, \mathrm{rSR}^{\prime}$ in lead V1 or V2, and S-waves in lead I and either lead V5 or V6. RBBB was further classified as having concomitant left anterior hemiblock (LAH) if the QRS axis was below $-30^{\circ}$ and concomitant left posterior hemiblock (LPH) if the QRS axis was above $90^{\circ} .{ }^{20}$ Discrepancies were resolved by consensus. Bifascicular block was defined as LBBB or RBBB with concomitant LAH or LPH.

\section{Statistical analyses and bioinformatics}

IBM SPSS V.20.0 (IBM Corp, Armonk, New York, USA) was used for statistical analyses. Continuous variables are expressed as mean $\pm \mathrm{SE}$ of the mean. Proportions are expressed as percentages. Allele frequencies were estimated from genotype data. The $\chi^{2}$ test was used to assess association per genotype and to evaluate deviations from the Hardy-Weinberg equilibrium. Testing for linkage disequilibrium (LD) was performed by the maximum likelihood method using the linkage utility program EH for estimation of haplotype frequencies. ${ }^{21}$ Haplotype effects on BBB and QRS were studied with the THESIAS program 3.1. ${ }^{22}$ Computer search for activation of potential transcription factor binding sites by the single nucleotide polymorphisms (SNPs) was performed with 
Table 2 Baseline characteristics and 12-lead ECG phenotype of the second cohort from the INTERGENE study

\begin{tabular}{|c|c|c|c|c|}
\hline & Controls $(n=248)$ & All BBB $(n=108)$ & LBBB $(n=35)$ & RBBB $(n=73)$ \\
\hline Mean age, years & $60.8 \pm 0.5$ & $63.7 \pm 0.8 \dagger$ & $63.9 \pm 1.2^{*}$ & $60.8 \pm 1.1^{*}$ \\
\hline Female, $\mathrm{n}(\%)$ & $92(37 \%)$ & $40(37 \%)$ & $15(43 \%)$ & 23 (33\%) \\
\hline $\mathrm{BMI}, \mathrm{kg} / \mathrm{m}^{2}$ & $26.7 \pm 0.2$ & $28.0 \pm 0.4 \dagger$ & $28.3 \pm 0.8^{\star}$ & $27.9 \pm 0.4^{\star}$ \\
\hline Systolic BP, mm Hg & $137.8 \pm 1.4$ & $140.0 \pm 1.9$ & $137.6 \pm 3.5$ & $141.1 \pm 2.2$ \\
\hline Diastolic BP, mm Hg & $83.6 \pm 0.6$ & $83.3 \pm 1.0$ & $79.7 \pm 1.8^{\star}$ & $85.0 \pm 1.2$ \\
\hline \multicolumn{5}{|l|}{ ECG phenotype } \\
\hline Heart rate, bpm & $63.7 \pm 0.6$ & $61.7 \pm 1.3$ & $62.3 \pm 2.3$ & $61.4 \pm 1.6$ \\
\hline$P Q$ & $166.8 \pm 1.4$ & $198.9 \pm 19.9^{*}$ & $181.9 \pm 4.4 \ddagger$ & $206.9 \pm 29.3^{*}$ \\
\hline QTc & $412.9 \pm 1.4$ & $453.8 \pm 2.8 \ddagger$ & $467.4 \pm 5.6 \ddagger$ & $447.3 \pm 2.8 \ddagger$ \\
\hline QRS duration, ms & $84.9 \pm 0.4$ & $138.7 \pm 1.4 \ddagger$ & $145.4 \pm 3.1 \ddagger$ & $135.6 \pm 1.4 \ddagger$ \\
\hline Q-axis & $+24.2 \pm 1.8$ & $-9.1 \pm 4.4 \ddagger$ & $-4.2 \pm 6.4 \ddagger$ & $-11.5 \pm 5.8 \ddagger$ \\
\hline \multicolumn{5}{|l|}{ History of } \\
\hline Myocardial infarction, n (\%) & $0(0 \%)$ & $44(41 \%) \ddagger$ & 19 (54\%)‡ & $25(34 \%) \ddagger$ \\
\hline Angina pectoris, n (\%) & $0(0 \%)$ & 39 (36\%)‡ & 12 (34\%)‡ & $27(37 \%) \ddagger$ \\
\hline Hypertension, $n(\%)$ & $44(18 \%)$ & $56(52 \%) \ddagger$ & $18(51 \%) \ddagger$ & $38(52 \%) \ddagger$ \\
\hline Diabetes, n (\%) & $11(4 \%)$ & $18(17 \%)$ & $9(26 \%)$ & $9(12 \%)^{\star}$ \\
\hline Hypercholesterolaemia, n (\%) & $31(13 \%)$ & $51(47 \%) \ddagger$ & $17(49 \%) \ddagger$ & $34(47 \%) \ddagger$ \\
\hline COPD, n (\%) & $14(6 \%)$ & $6(6 \%)$ & $1(3 \%)$ & $5(7 \%)$ \\
\hline Ever a regular smoker, $\mathrm{n}(\%)$ & $137(55 \%)$ & $71(66 \%)$ & $2(71 \%)$ & $46(63 \%)$ \\
\hline Arthritis, n (\%) & $8(3 \%)$ & $6(6 \%)$ & $2(6 \%)$ & $4(6 \%)$ \\
\hline Irregular pulse, n (\%) & $15(6 \%)$ & $22(20 \%) \ddagger$ & $10(29 \%) \ddagger$ & $12(16 \%) \dagger$ \\
\hline
\end{tabular}

SNPInspector, searching the MatInspector database. ${ }^{23}$ Computer search for potential influence on messenger RNA (mRNA) stability was performed with UTRdb. ${ }^{24}$ LD between the identified polymorphism at the connexin 43 locus and the SNP rs1015451 identified by previous genome-wide association studies was performed by PLINK. ${ }^{25}$

\section{Missing data}

In the association analysis, one patient had missing data for the connexin 40679 genotype and one patient for the connexin $431400 \mathrm{~A}$ /insertion genotype.

\section{Ethics statement}

The protocol was approved by the ethical board of the University of Gothenburg, and patients provided written informed consent.

\section{RESULTS}

\section{Step 1: heredity}

Two sons with complete BBB were identified $(6.4 \%$ of all participating men). The only complete RBBB was in a 56-year-old son with a RBBB with QRS duration of 132 ms. His father had had an RBBB with QRS duration of $135 \mathrm{~ms}$ when measured at age 67. No BBB or disturbances in ECG measurements were found in the daughters (table 1). None of the two sons with complete BBB had any symptoms or ECG findings indicative of ischaemic heart disease or heart failure.

\section{Step 2: resequencing}

By resequencing connexin 40, six SNPs were identified at positions $-992,-679,-55,+197,+312$ and +2469 relative to transcription start and the resulting mRNA in GenBank (BC013313). All SNPs were in Hardy-Weinberg equilibrium. Frequencies and corresponding rs numbers

Table 3 Sequenced single nucleotide polymorphisms (SNPs) at the connexin 40 locus

\begin{tabular}{llll}
\hline Polymorphism & rs number & Sequence & Frequency \\
\hline Cx40 992G>C & rs791275 & CCTCAAGACCTG[C/G]ATGGGTCTTTGA & 0.56 \\
Cx40-679C $>$ T & rs79030318 & AACCTCAGATTT[A/G]GGTGGGTCCTTA & 0.87 \\
Cx 40-55A>G & rs10465885 & AGGTGTTTAATA[C/T]ATCTTAGTTGAG & 0.53 \\
Cx40 197T>C & rs12136839 & ACATGTCCCCTC[A/G]CCCCTAGCTGAA & 0.95 \\
Cx40 312A>G & rs6593809 & AATCCTCCCTCA[C/T]CTGGCCTTGTC & 0.69 \\
Cx40 2469G $>A$ & rs1692141 & GACACGTCTTTC[C/T]TCTCTGAGCCTC & 0.78 \\
\hline Cx, connexin. & & &
\end{tabular}


Table 4 Pairwise linkage disequilibrium coefficients $\left(D^{\prime}\right)$ between polymorphisms at the connexin 40 locus

\begin{tabular}{|c|c|c|c|c|c|c|}
\hline Polymorphism & Cx40-992 & Cx40-679 & $\begin{array}{l}D^{\prime} \\
\text { Cx40-55 }\end{array}$ & Cx40 197 & Cx40 312 & Cx40 2469 \\
\hline Cx40-992 & & 0.62 & 1 & 1 & 0.93 & 0.89 \\
\hline Cx40-679 & & & 1 & 1 & 1 & 0.75 \\
\hline Cx40-55 & & & & 1 & 1 & 0.69 \\
\hline Cx40 197 & & & & & 1 & 0.79 \\
\hline Cx40 312 & & & & & & 0.97 \\
\hline Cx40 2469 & & & & & & \\
\hline
\end{tabular}

are shown in table 3 and linkage equilibrium in table 4 . Searching for putative transcription factor binding sites using SNPInspector revealed that although the connexin 40 SNPs altered transcription factor binding sites for sites located in the connexin 40 promoter $(-992,-679$, $-55)$ and in the first intron $(+197,+312)$, none of the listed transcription factors has been shown to be present in the heart (SNPInspector website search, 4 June 2014). The connexin $402469 \mathrm{G}>\mathrm{A}$ SNP was located in the $3^{\prime}$ flanking region, $56 \mathrm{bp}$ downstream of last amino acid, and did not affect mRNA stability according to UTRdb.

Resequencing of connexin 43 resulted in the identification of only one polymorphism, which was a single nucleotide insertion/deletion polymorphism in the noncoding part of the second exon at position 1400 relative to transcription start and resulting mRNA in GenBank (NM_000165.3). The connexin 43 1400A/ins was in Hardy-Weinberg equilibrium, had a minor allele frequency of 0.04 and is annotated rs67678923. The inserted $\mathrm{A}$ is inserted immediately after the coding sequence in the first nucleotide position in the 3'-untranslated region (UTR).

\section{Step 3: Association}

LBBB was more evenly distributed among men and women, whereas RBBB was more common in men (table 2). Hypertension, angina pectoris and hypercholesterolaemia were equally distributed among LBBB and $\mathrm{RBBB}$ and, as expected, they were more prevalent in these subjects than in the control group without cardiovascular disease. Subjects with LBBB more often had a history of myocardial infarction, diabetes and irregular pulse compared to the control group but also compared to subjects with RBBB (table 2).

Genotype distribution of the connexin $431400 \mathrm{~A} / \mathrm{ins}$ polymorphism was associated with LBBB $\left(\chi^{2}=7.3, p=0.03\right)$ but not with RBBB, and thus not with total BBB (table 5). Owing to the frequency of the connexin 43 variant, a recessive model cannot be tested in the present material (only one subject homozygous for the variant). Testing with allelic OR is also hampered by low numbers. In a dominant model OR is 1.87 (0.59-5.97), $\beta=0.626$, $\mathrm{p}=0.29$. If adjusted for age and sex the $\mathrm{OR}$ is 1.95 (0.60-6.32), $\beta=0.67, p=0.26$. When analysing the connexin
43 with QRS duration as a continuous variable, no significant difference was observed, although the single homozygous individual for connexin 43 1400A/ins polymorphism had a very long QRS duration.

None of the individually identified SNPs in connexin 40 were associated with $\mathrm{BBB}$, either LBBB, RBBB or total BBB (table 5).

Based on connexin 40 genotype data and haplotypes of the screening panel, these haplotypes were tagged by four SNPs in the INTERGENE cohort. Four haplotypes occurred with a frequency of over $5 \%$ and these haplotypes corresponded to $95 \%$ of alleles; there were seven haplotypes with a frequency over $1 \%$ (table 6 ).

To further explore any relation of conduction and genetic variation at the connexin 40 locus, we performed a regression analysis of QRS duration as a continuous variable and haplotypes of connexin 40 . Haplotype analysis of connexin 40 showed no relation to QRS duration. Individually assigned haplotypes of connexin 40 and QRS duration are displayed in figure 2.

\section{DISCUSSION}

The association of LBBB and connexin 43 1400A/ins polymorphism together with this and previous studies on heritability, indicate that BBB development can be modulated by genetic factors and could be the result of a progressive disorder within the myocardium and not confined to the specialised conduction system.

In our study on heritability, we report the first study focused on BBB in fathers and their descendants in their 50s. From our previous study of the fathers, The Study of Men Born 1913, ${ }^{3}$ we know that at age 50 the prevalence of BBB among men is $1.2 \%$. The sons had a longer QRS duration compared with the daughters. This, and the finding that two $(6.4 \%)$ of the sons also had BBB, but none of the daughters did, might indicate a male predominance of BBB. Previous studies have shown a male predominance of RBBB but not for LBBB. ${ }^{26}$ The male predominance of $\mathrm{BBB}$ may be a paraphenomenon with cardiovascular disease, in keeping with the fact that women generally develop cardiovascular disease later than men; alternatively, it may represent a sex-linked inheritance of a conduction disorder unrelated to cardiovascular disease. Unfortunately, the present study cannot 
Table 5 Bundle branch blocks (BBBs) versus polymorphisms in connexin 40 and connexin 43

\section{Genotype}

BBB (n)

No BBB (n)

p Value

(A) $B B B$ vs no $B B B$

Cx40-992

Cx40-679

GG $\quad 39$

77

121

50

191

52

$n=373$

CC

53

79

4

60

Cx40-55

TT

$\mathrm{n}=374$

GG 21

Cx40 2469

$\mathrm{GA}$

AA

$\mathrm{n}=374$

GG

GA

Cx43 1400

AA 6

$\mathrm{n}=373$

AA 95

A/- 11

-l- 1

(B) RBBB vs no $B B B$

Cx40-992 GG 24

$\mathrm{n}=374$

GG

Cx40-679

CC 52

$\mathrm{n}=373$

CT 18

TT 3

Cx40-55

$\mathrm{n}=374$

GG

$\mathrm{GA}$

$A A$

Cx40 2469

$\mathrm{n}=374$

$\mathrm{Cx} 0431400$
$\mathrm{n}=373$

GA 21

AA 4

AA 64

A/- 8

(C) $L B B B$ vs no $B B B$

Cx40-992 GG

$\mathrm{n}=374$

GG 15

Cx40-679

$\mathrm{n}=373$

GC 16

CC 4

CC 27

CT 8

TT 0

Cx40-55

$\mathrm{n}=374$

Cx40 $2469 \quad$ AA 8

$\mathrm{n}=374$

Cx43 1400

$\mathrm{n}=373$

GA 13

AA 2

AA 31

A/- 3

-l- 1

(D) Bifascicular block vs no BBB

$\begin{array}{llrr}\mathrm{C} \times 40-992 & \text { GG } & 21 & 77 \\ \mathrm{n}=374 & \text { GC } & 32 & 121 \\ & \text { CC } & 9 & 50 \\ \mathrm{C} \times 40-679 & \mathrm{CC} & 48 & 191 \\ \mathrm{n}=373 & \mathrm{CT} & 13 & 52 \\ & \text { TT } & 1 & 4 \\ \mathrm{C} \times 40-55 & \mathrm{GG} & 12 & 60 \\ \mathrm{n}=374 & \mathrm{GA} & 32 & 116\end{array}$

0

77

121

50

191

52

4

60

116

72

146

88

14

232

16

0

77

121

50

191

52

4

60

116

72

146

88

14

232

16

0

77

121

50

52

4

16
Table 5 Continued

\begin{tabular}{lllll}
\hline Genotype & & BBB (n) & No BBB (n) & p Value \\
\hline \multirow{4}{*}{ Cx40 2469 } & AA & 18 & 72 & \\
$\mathrm{n}=374$ & GG & 43 & 161 & 0.31 \\
& GA & 16 & 88 & \\
Cx43 1400 & AA & 3 & 14 & \\
$\mathrm{n}=373$ & AA & 56 & 232 & 0.12 \\
& A/ & 5 & 16 & \\
\hline p Values for $\chi^{2}$ test for each genotype. \\
Cx, connexin; LBBB, left BBB; RBBB, right BBB.
\end{tabular}

0.42

0.61

0.55

0.87

Continued give us the answer to this question. Although not focused on BBB, previous twin and family studies have reported a heritability of QRS duration of $0.36-0.43 .^{27} 28$

We chose a candidate gene approach to explore the association between heritable factors and QRS duration. On the basis of knock-out mice and functional studies, ${ }^{9}{ }^{11}$ we chose connexin 40 and connexin 43 . With our extreme phenotypes approach, we identified 6 SNPs in connexin 40 and 1 insertion/deletion polymorphism in connexin 43. This is lower than expected, despite the fact that the present study had reasonable power (over $99 \%$ for biallelic variants with a frequency above $10 \%$ ). Our screening approach with extreme phenotypes might influence the number of identified variants, as we only intended to identify plausible functional SNPs associated with QRS width. It is likely that a random sample might identify more genetic variants.

The finding of connexin 43 and the association to LBBB is supported by genome-wide association studies (GWAS) as well as by functional data. In a large GWAS, genetic markers close to the gene for connexin 43, GJA1, showed a strong association with QRS duration. ${ }^{29}$ The allele frequency for our identified connexin 43 polymorphism, rs67678923, and the strongest marker of the GJA1 locus in the GWAS, rs1015451, differs, and therefore the linkage expressed as $\mathrm{R}^{2}$ and $\mathrm{D}^{\prime}$ differs; using PLINK in 1000 Genomes project (1092 individuals in phase 1$), \mathrm{R}^{2}$ was 0.02 and $\mathrm{D}^{\prime}$ was $0.89 .^{25}$

From a functional point of view, there is a growing body of evidence that connexin 43 is a major player in cardiac conduction. ${ }^{14}{ }^{30}$ Connexin 43 can be regulated at the post-transcriptional level, thereby affecting connexin 43 turnover and function. ${ }^{30}$ Ischaemia induces dephosphorylation of connexin 43 and its translocation from the gap junction to intracellular stores, with reduced conduction as a result. ${ }^{31}$ The connexin 43 insertion/deletion polymorphism (rs144617620) has an inserted A immediately after the coding sequence in the first nucleotide position in the $3^{\prime}$-UTR. The association of connexin 43 polymorphism and LBBB might be explained by post-translational regulation of connexin 43 with reduced mRNA stability and connexin 43 levels, and thereby conduction. Functional characterisation of the identified connexin 43 polymorphism would of 
Table 6 Haplotypes of connexin 40

\begin{tabular}{llllll}
\hline Haplotype & $-992 G>C$ & $-679 C>T$ & $-55 A>G$ & 2469G $>$ A & Frequency \\
\hline H1 & C & C & A & G & 0.42 \\
H2 & G & C & G & G & 0.23 \\
H3 & G & C & G & A & 0.19 \\
H4 & G & T & A & G & 0.09 \\
H5 & G & C & G & A & 0.04 \\
H6 & C & C & A & G & 0.02 \\
H7 & & & & $G$ & 0.01 \\
\hline
\end{tabular}

course strengthen our finding and minimise the risk that it only represents a chance finding or is in LD with a functional variant. However, such an analysis is beyond the scope of the present paper, and should be carried out in separate studies.

Genetic variation at the connexin 40 locus has previously been shown to be associated with atrial fibrillation, but not with BBB or QRS duration. ${ }^{32}$ As these SNPs were not associated with our phenotype of interest, their further characterisation is beyond the scope of this paper.

Whether to study BBB as a group or RBBB and LBBB as separate entities is a matter of dispute. Most studies looking at LBBB and RBBB as separate entities are based on hospitalised patients; the majority have ischaemic heart disease where RBBB is more frequent than LBBB, due to myocardial scarring after myocardial infarction. ${ }^{33}{ }^{34}$ Data from our epidemiological study ${ }^{3}$ suggest that BBB develops gradually. We hypothesised that this could be due to a progressive conduction disorder and decided to use a genetic approach without any assumptions about whether this could affect LBBB, RBBB or all types of BBB.

The present paper has some strengths and weaknesses. As shown by our previous study, BBB is a late-onset phenotype. ${ }^{3}$ The access to 80 -year-old men without BBB represents true controls for people without BBB, which is hard to get. For the same reason, it is possible that the

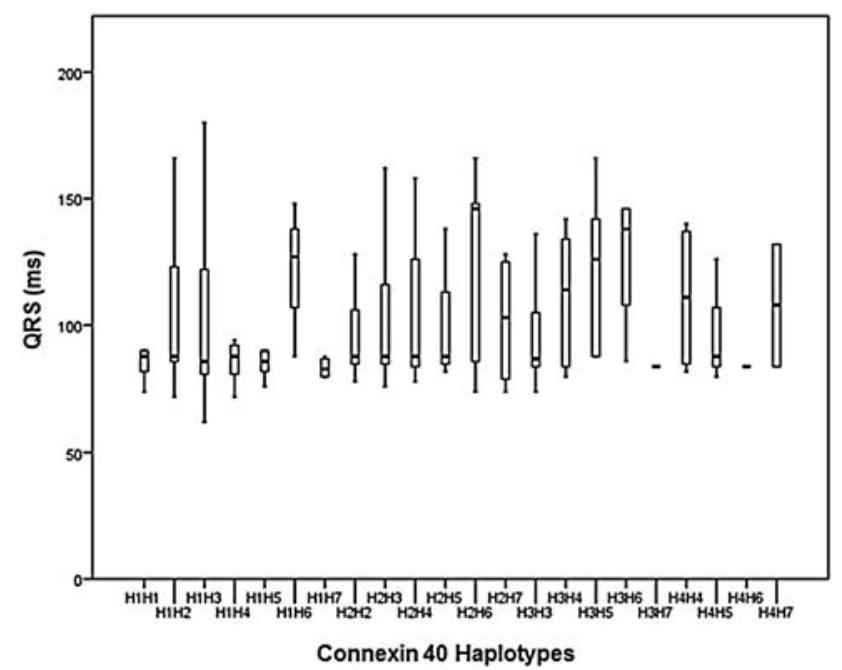

Figure 2 Lack of association of QRS duration with individually assigned connexin 40 haplotypes. adult descendants in the heritability study were too young to develop BBB. Reassessment at an older age would probably increase the probability of finding more BBBs among them. In this study we only had ECG information for the fathers, but data for both parents would improve heritability assessment, especially on X linked traits. Regarding the association study, the INTERGENE study was a case-control study on ischaemic heart disease. Controls in the present study were derived from the population sample without prior cardiovascular disease and were thus less likely to have cardiovascular risk factors. The high prevalence of ischaemic heart disease and its risk factors in the present study does not necessarily reflect the true prevalence in people with or without BBB in the general population. Moreover, although a specific hypothesis was tested in this small study, the finding of connexin 43 polymorphism associated with LBBB must be replicated in larger studies with better power.

In conclusion, $\mathrm{BBB}$ is the result of a prolonged conduction within the heart. This can be caused by acute ischaemic lesions to the conduction system, that is, myocardial infarction. The heritability of longer QRS duration, and the association of LBBB and connexin 43 polymorphism, indicate that it can also be caused by a progressive conduction disorder within the myocardium distal to the specialised conduction system.

Acknowledgements The authors thank research nurse Helena Dellborg for assistance in the descendants study. We thank Cecilia Lundholm for technical assistance in the sequencing and genotyping study. They also thank the Genomics Core Facility at the Sahlgrenska Academy, University of Gothenburg, for providing genotyping facilities.

Contributors PL drafted the article, designed the study including genetic analysis and performed statistical analyses. BA designed and performed genetic analyses and bioinformatics. P-OH and HE were responsible for the 1913 study. DT was responsible for the INTERGENE study. MD interpreted ECGs. PE designed the study, was responsible for the descendants study and interpreted ECGs. All authors were involved in the interpretation of results and reviewing the manuscript before submission.

Funding The study was funded by grants from the Swedish state under the LUA/ALF agreement.

Competing interests None.

Ethics approval Ethical board of Gothenburg University.

Provenance and peer review Not commissioned; externally peer reviewed.

Data sharing statement Primer and probe data are available to readers on request by email to the corresponding author. 
Open Access This is an Open Access article distributed in accordance with the Creative Commons Attribution Non Commercial (CC BY-NC 4.0) license, which permits others to distribute, remix, adapt, build upon this work noncommercially, and license their derivative works on different terms, provided the original work is properly cited and the use is non-commercial. See: http:// creativecommons.org/licenses/by-nc/4.0/

\section{REFERENCES}

1. Kumar V, Venkataraman R, Aljaroudi W, et al. Implications of left bundle branch block in patient treatment. Am J Cardiol 2013;111:291-300.

2. Breithardt $\mathrm{G}$, Breithardt $\mathrm{OA}$. Left bundle branch block, an old-new entity. J Cardiovasc Trans/ Res 2012;5:107-16.

3. Eriksson $\mathrm{P}$, Hansson $\mathrm{PO}$, Eriksson $\mathrm{H}$, et al. Bundle-branch block in a general male population: the study of men born 1913. Circulation 1998:98:2494-500.

4. Aro AL, Anttonen O, Tikkanen JT, et al. Intraventricular conduction delay in a standard 12-lead electrocardiogram as a predictor of mortality in the general population. Circ Arrhythm Electrophysiol 2011;4:704-10.

5. Schneider JF, Thomas HE Jr, Kreger BE, et al. Newly acquired left bundle-branch block: the Framingham study. Ann Intern Med 1979;90:303-10.

6. Schneider JF, Thomas HE, Kreger BE, et al. Newly acquired right bundle-branch block: the Framingham study. Ann Intern Med 1980;92:37-44.

7. Risum N, Strauss D, Sogaard P, et al. Left bundle-branch block: the relationship between electrocardiogram electrical activation and echocardiography mechanical contraction. Am Heart $J$ 2013;166:340-8.

8. Sipahi I, Chou JC, Hyden M, et al. Effect of QRS morphology on clinical event reduction with cardiac resynchronization therapy: meta-analysis of randomized controlled trials. Am Heart $J$ 2012;163:260-7.e3.

9. Kirchhoff S, Kim JS, Hagendorff A, et al. Abnormal cardiac conduction and morphogenesis in connexin40 and connexin43 double-deficient mice. Circ Res 2000;87:399-405.

10. Dupont E, Matsushita T, Kaba RA, et al. Altered connexin expression in human congestive heart failure. $\mathrm{J} \mathrm{Mol}$ Cell Cardiol 2001;33:359-71.

11. Fontes MS, van Veen TA, de Bakker JM, et al. Functional consequences of abnormal Cx43 expression in the heart. Biochim Biophys Acta 2012;1818:2020-9.

12. Simon AM, Goodenough DA, Paul DL. Mice lacking connexin 40 have cardiac conduction abnormalities characteristic of atrioventricular block and bundle branch block. Curr Biol 1998;8:295-8.

13. Tamaddon HS, Vaidya D, Simon AM, et al. High-resolution optical mapping of the right bundle branch in connexin40 knockout mice reveals slow conduction in the specialized conduction system. Circ Res 2000;87:929-36.

14. Jansen JA, van Veen TA, de Bakker JM, et al. Cardiac connexins and impulse propagation. J Mol Cell Cardiol 2010;48:76-82.

15. Tibblin G, Aurell E, Hjortzberg-Nordlund $\mathrm{H}$, et al. A general health-examination of a random sample of 50 -year-old men in goeteborg. Acta Med Scand 1965;177:739-49.

16. Berg CM, Lissner L, Aires N, et al. Trends in blood lipid levels, blood pressure, alcohol and smoking habits from 1985 to 2002: results from INTERGENE and GOT-MONICA. Eur J Cardiovasc Prev Rehabil 2005;12:115-25.
17. Ladenvall $P$, Wall $U$, Jern $S$, et al. Identification of eight nove single-nucleotide polymorphisms at human tissue-type plasminogen activator (t-PA) locus: association with vascular t-PA release in vivo. Thromb Haemost 2000;84:150-5.

18. Gunnarsson G, Eriksson P, Dellborg M. ECG criteria in diagnosis of acute myocardial infarction in the presence of left bundle branch block. Int J Cardiol 2001:78:167-74.

19. Surawicz B, Childers R, Deal BJ, et al. AHA/ACCF/HRS recommendations for the standardization and interpretation of the electrocardiogram: part III: intraventricular conduction disturbances: a scientific statement from the American Heart Association Electrocardiography and Arrhythmias Committee, Council on Clinical Cardiology; the American College of Cardiology Foundation; and the Heart Rhythm Society: endorsed by the International Society for Computerized Electrocardiology. Circulation 2009;119: e235-240.

20. Gunnarsson G, Eriksson P, Dellborg M. Continuous ST-segment monitoring of patients with right bundle branch block and suspicion of acute myocardial Infarction. Ann Noninvasive Electrocardiol 2005;10:161-8.

21. Terwilliger J, Ott J. Linkage disequilibrium between alleles at marker loci. In: Terwilliger J, Ott J, eds. Handbook of human genetic linkage. Baltimore: John Hopkins University Press, 1994:188-98.

22. Tregouet DA, Escolano S, Tiret L, et al. A new algorithm for haplotype-based association analysis: the Stochastic-EM algorithm. Ann Hum Genet 2004;68:165-77.

23. Cartharius K, Frech K, Grote K, et al. Matlnspector and beyond: promoter analysis based on transcription factor binding sites. Bioinformatics 2005;21:2933-42.

24. Grillo G, Turi A, Licciulli F, et al. UTRdb and UTRsite (RELEASE 2010): a collection of sequences and regulatory motifs of the untranslated regions of eukaryotic mRNAs. Nucleic Acids Res 2010;38:D75-80.

25. Purcell S, Neale B, Todd-Brown K et al. PLINK: a tool set for whole-genome association and population-based linkage analyses. Am J Hum Genet 2007;81:559-75.

26. De Bacquer D, De Backer G, Kornitzer M. Prevalences of ECG findings in large population based samples of men and women. Heart 2000;84:625-33.

27. Mutikainen S, Ortega-Alonso A, Alen M, et al. Genetic influences on resting electrocardiographic variables in older women: a twin study. Ann Noninvasive Electrocardiol 2009;14:57-64.

28. Li J, Huo Y, Zhang Y, et al. Familial aggregation and heritability of electrocardiographic intervals and heart rate in a rural Chinese population. Ann Noninvasive Electrocardiol 2009;14:147-52.

29. den Hoed M, Eijgelsheim M, Esko T, et al. Identification of heart rate-associated loci and their effects on cardiac conduction and rhythm disorders. Nat Genet 2013;45:621-31.

30. Klotz LO. Posttranscriptional regulation of connexin-43 expression. Arch Biochem Biophys 2012;524:23-9.

31. Beardslee MA, Lerner DL, Tadros PN, et al. Dephosphorylation and intracellular redistribution of ventricular connexin43 during electrical uncoupling induced by ischemia. Circ Res 2000;87:656-62.

32. Firouzi M, Ramanna H, Kok B, et al. Association of human connexin40 gene polymorphisms with atrial vulnerability as a risk factor for idiopathic atrial fibrillation. Circ Res 2004;95:e29-33.

33. Strauss DG, Loring $\mathrm{Z}$, Selvester $\mathrm{RH}$, et al. Right, but not left, bundle branch block is associated with large anteroseptal scar. J Am Coll Cardiol 2013;62:959-67.

34. Scheinman M, Brenman B. Clinical and anatomic implications of intraventricular conduction blocks in acute myocardial infarction. Circulation 1972;46:753-60. 